\title{
Análisis de indicadores productivos y económicos en la hacienda bananera san alfonso, cantón el guabo
}

\section{Analysis of productive and economic indicators in the hacienda bananera san alfonso, cantón el guabo}

DOI: $10.46932 / s f j d v 2 n 4-056$

Received in: May 1st, 2021

Accepted in: Jun 30th, 2021

\author{
Denisse Margarita Vera Urbina \\ Estudiante carrera de economía agropecuaria \\ Universidad Técnica de Machala. Km 5 1/2 vía Pasaje \\ Machala-Ecuador \\ E-mail: dvera3@utmachala.edu.ec \\ Jessica Maribel Quezada Campoverde \\ Ingeniera agrónoma \\ Universidad Técnica de Machala. Km 5 1/2 vía Pasaje \\ Machala-Ecuador \\ E-mail: jquezada@utmachala.edu.ec \\ Eveligh Prado-Carpio \\ $\mathrm{PhD}$. \\ Ingeniera comercial \\ Universidad Técnica de Machala. Km 5 1/2 vía Pasaje \\ Machala-Ecuador \\ E-mail: eprado@utmachala.edu \\ Víctor Javier Garzón Montealegre \\ Economista \\ Universidad Técnica de Machala. Km 5 1/2 vía Pasaje \\ Machala-Ecuador \\ E-mail:vgarzon@utmachala.edu.ec
}

\section{RESUMEN}

La actividad bananera está estrechamente ligada en el desarrollo económico y social del país, por ello es indispensable conocer los factores que influyen y las falencias en el proceso productivo para intervenir con posibles soluciones que permitan aumentar en su productividad e incrementar la rentabilidad, el presente trabajo tiene como objetivo analizar los indicadores productivos y económicos en la producción de banano Cavendish de la Hacienda San Alfonso, ubicada en el sitio la Bocatoma del cantón El Guabo, bajo un diseño no experimental de campo y corte transversal con enfoque descriptivo-explicativo, lo que conlleva a identificar los indicadores principales dentro de la producción, relacionar su influencia en la rentabilidad y comparar la productividad entre los periodos 2018-2019, con la finalidad de determinar el año más productivo dentro de los intervalos mencionados. La investigación determinó que el año 2018 tuvo mayor productividad agrícola asociados con la producción y los factores productivos, como son las cajas obtenidas por medio de la eficiencia y calidad del fruto con base al peso, obteniendo como resultado un peso de fruta exportable de 79,31\%, del cual se genera 94.295 cajas de banano, representando un ingreso económico de $\$ 587.425,53$ y una utilidad positiva de $\$ 33.565,94$. 
Palabras Claves: Banano, indicadores productivos, indicadores económicos, rentabilidad.

\begin{abstract}
The banana activity is closely linked to the economic and social development of the country, therefore it is essential to know the factors that influence and the shortcomings in the production process to intervene with possible solutions to increase productivity and increase profitability, this work aims to analyze the productive and economic indicators in the banana production of the Hacienda San Alfonso located in the site of the Bocatoma of the canton El Guabo, under a non-experimental field and cross-sectional design with a descriptive-explanatory approach, which leads to identify the main indicators within the production, relate their influence on profitability and compare productivity between the periods $2018-2019$, in order to determine the most productive year within the mentioned intervals. The research determined that 2018 had higher agricultural productivity associated with production and productive factors, such as boxes obtained through efficiency and fruit quality based on weight, resulting in an exportable fruit weight of $79.31 \%$, from which 94,295 banana boxes are generated, representing an economic income of $\$ 587,425.53$ and a positive profit of $\$ 33,565.94$.
\end{abstract}

Keywords: Banana, production indicators, economic indicators, profitability.

\title{
1 INTRODUCCIÓN
}

Según FAO (2020), la producción mundial de banano en los periodos 2017-2019 fue de 116 millones ${ }^{\mathrm{TM}}$, equivalente a los 31.000 millones de dólares americanos, se producen alrededor de 1.000 variedades, pero apenas el $15 \%$ se comercializa, observe en la figura 1, el volumen de exportación a nivel mundial en toneladas métricas por región.

Figura 1. Exportación mundial de banano por región.

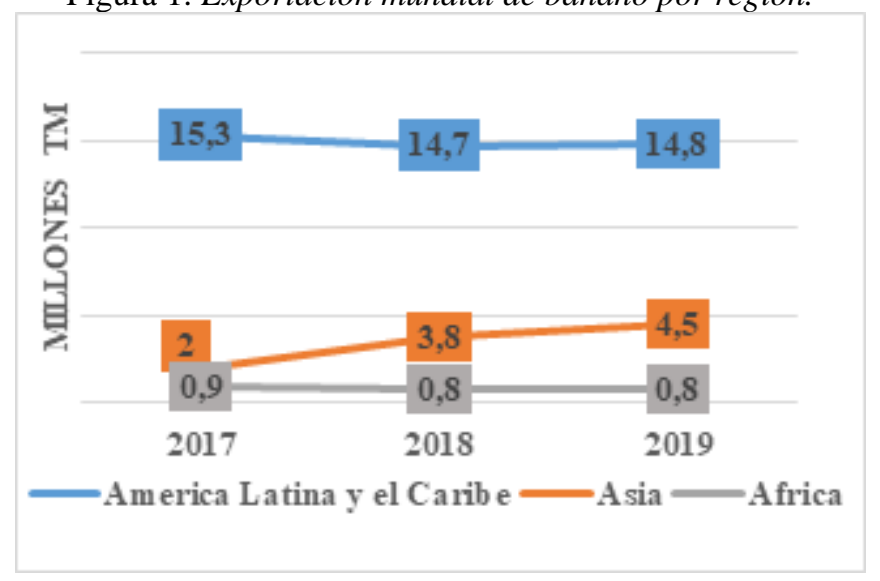

Fuente: FAO 2020.

En la Figura 1, se observa que la mayor parte de banano exportado en el mundo proviene de los países de América Latina y el Caribe, en el año 2017 alcanza el récord más alto obteniendo 15.3 millones Tm, se estimó una tendencia alcista para el siguiente año, sin embargo, sufre una caída del 4\%, provocado 
por el fenómeno natural de El Niño en Costa Rica, quien lidera en volúmenes de producción, por ende, el notable descenso que perdura en el año 2019, quien tiene un nivel de rendimiento similar.

FAO (2020), En el primer semestre del 2020 la exportación mundial alcanzó un nuevo máximo de 12.2 millones $^{\mathrm{Tm}}$, en relación al primer semestre del año anterior debido al impacto económico y social ocasionado por la pandemia de la COVID-19, los indicadores apuntan a una recesión por las perturbaciones experimentadas en el mercado.

Según INEC (2020), Ecuador es el número uno en exportación de banano a escala mundial, el rendimiento de la actividad bananera está compuesta por pequeños, medianos y grandes productores, quienes participan con el $12.34 \%$ en superficie total plantada dentro de los cultivos permanentes, a medida que pasa el tiempo el volumen de producción incrementa debido a la competitividad con el fin de abastecer la demanda en el mercado exterior.

El banano ecuatoriano se exporta a más de 15 países, los principales consumidores son: Rusia, Estados Unidos, China e Italia, al ser considerado como el primer proveedor a nivel internacional, las exportaciones del producto ocupan el segundo lugar, luego del petróleo, siendo una de las fuentes más importantes en la economía del país (García, et al., 2016).

La producción de banano genera alrededor de 2.5 millones plazas de empleo de forma directa e indirecta, aporta al Producto Interno Bruto un 2\% y un 35\% al PIB agropecuario, la calidad del producto le ha permitido ubicarse como el exportador principal, cada año se exporta aproximadamente 300 millones de cajas por todo el mundo, el cual le proporciona tener un desarrollo económico y a su vez un desarrollo social, véase en la Tabla 1, la producción nacional en los periodos 2017-2020.

Tabla 1. Producción de banano nacional.

\begin{tabular}{lccccc}
\hline & $\left(\mathrm{Ha}^{-1}\right)$ & 2017 & 2018 & 2019 & 2020 \\
\hline Superficie $\left(\mathrm{Ha}^{-1}\right)$ & Plantada & 166 & 173 & 190 & 165 \\
\cline { 2 - 5 } & Cosechada & 158 & 161 & 183 & 160 \\
\hline \begin{tabular}{l} 
Prod. ('M) \\
\cline { 2 - 5 }
\end{tabular} & & $6^{\prime} 282.105$ & $6^{\prime} 506.635$ & $6^{\prime} 583.477$ & $6^{\prime} 023.390$ \\
\hline $\begin{array}{l}\text { Prod. vendida } \\
\text { (TM) }\end{array}$ & $6^{\prime} 506.309$ & $6^{\prime} 413.259$ & $6^{\prime} 513.549$ & $5^{\prime} 917.569$ \\
\hline
\end{tabular}

Fuente: INEC (2021)

En el país existe 3 provincias con mayor volumen de producción, entre ellos la provincia de los Ríos, lidera con el 36\%, le sigue Guayas con el 29\% y El Oro se sitúa en el tercer puesto de la escala productiva con el $25 \%$, cuenta con el $45 \%$ de número de productores quienes poseen fincas inferiores a 5 
$\mathrm{ha}^{-1}$, mientras que los grandes productores se encuentran en Guayas representado por el 34\% y los Ríos por el 16\%, debido a su mayor extensión (Chungata, et al., 2019).

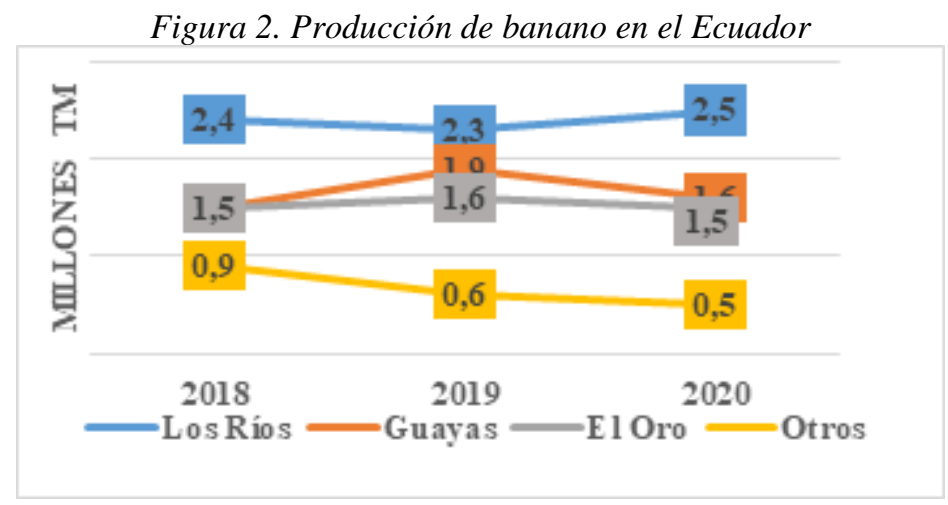

Fuente: INEC (2021)

En la Figura 2. Se visualiza el rendimiento de la producción de banano de los últimos 3 años, en el año 2019 alcanza los 6’6 mil Tm, mientras que en el año 2020 decrece a 6’1 mil ${ }^{\mathrm{Tm}}$, provocado por la COVID-19, alrededor del 22\% de la producción fue enviado al mercado ruso (BCE, 2019).

La investigación tiene como objetivo principal analizar los indicadores productivos y económicos en la producción de banano de la Hacienda San Alfonso del productor Agrícola el Cocoke S.A., con el fin de determinar su influencia en la productividad y rentabilidad entre los periodos 2018-2019.

\section{DESARROLLO}

El banano se caracteriza por ser una planta monocotiledónea, herbácea y perenne, su origen se da en el sudeste de Asia y sus primeros indicios datan en Papúa Nueva Guinea en el siglo VII a.C., ha sido uno de los primeros alimentos del hombre primitivo, debido a que han sido cultivadas desde aproximadamente 10.000 años, su expansión a nivel mundial se debe a la migración poblacional provocando cruces naturales que diversifican su genética (Lassoudière, 2010).

Según la Organización de las Naciones Unidas para la Alimentación y la Agricultura (FAO), la producción de banano a nivel mundial mantiene una tendencia al alza debido al aumento de productores quienes buscan elevar los volúmenes de productividad y cumplir con las exigencias del sector bananero apuntando al mercado internacional, el cultivo se encuentra ubicado en el quinto lugar como uno de los frutos más importantes en el mundo, después del trigo, arroz, maíz y papa.

En América Latina y el Caribe es considerado un cultivo rentable puesto que las condiciones climáticas permiten producir a gran escala, el aumento de la producción lo convierten en el cultivo más extenso, aunque también se produce en África, Asia e Islas Canarias del continente europeo, sin embargo la escala es inferior, las variedades Gros Michel, Valery y Cavendish cuentan con una alta tasa de 
comercialización (Zhiminaicela, et al., 2020), sin embargo, la serie Cavendish es la más consumida por su calidad, (Prado et al., 2021), manifiestan que la calidad consiste en cumplir con las cualidades requeridas por el cliente, como el buen sabor, textura y otros, a ello, se debe el aumento considerado en volúmenes de producción cada año.

De América Latina el Ecuador es el mayor exportador de banano y a nivel mundial y líder desde hace 25 años, su producción empieza desde 1910, pero no es hasta en 1952 que comienza la verdadera comercialización (Chica, et al., 2020), en el transcurso del tiempo experimenta un crecimiento exponencial asociado con la fertilidad del suelo, el clima idóneo para las plantaciones y la intervención del gobierno a cargo de Galo Plazo Lasso, quien se dedicó a fortalecer el comercio por las aportaciones en el producto interno bruto de la actividad agrícola, lo cual mejora la estructura socioeconómica en el país (Vásquez, et al., 2019).

Según el informe del Ministerio de Agricultura y Ganadería (MAG), en el Ecuador existen 4.473 productores dedicados a la actividad bananera y alrededor del $95.6 \%$ está conformado por pequeños y medianos productores (Ministerio de Comercio Exterior, 2017), lo cual inciden en la productividad y provoca que el país no lidere en volúmenes de producción, a nivel mundial se encuentra en el quinto puesto del ranking con mayor rendimiento, por lo tanto, existe una constante competencia, principalmente contra Costa Rica, Colombia y otros países Latino Americanos (Galarza, 2019).

El aporte al sector productivo que brinda el Gobierno Nacional a través del Ministerio de Agricultura y Ganadería (MAG), tiene como objetivo promover el desarrollo económico mediante un plan de inversión y renovación en las plantaciones de banano, lo cual permite explotar a mayor escala el cultivo, reducir los costos, generar mayor rentabilidad y reducir la brecha frente a otros países productores, incluye la asistencia técnica para capacitar a los productores y al personal bajo el manual de seguridad y salud e incentivar el uso de protección personal con la finalidad de obtener un mejor manejo y evitar riesgos laborales.

Según Vital (2017), cuando los trabajadores no hacen uso de los medios de protección durante cada actividad ponen en riesgo su salud y bienestar físico, ya sea por la carencia de recursos, falta de conocimiento o comodidad al momento de desempeñar las siguientes labores culturales:

- Cirugía de hoja: Consiste en retirar las partes afectadas por la Sigatoka.

- Descapote: Se procede a quitar las vainas de todas las hojas secas del racimo.

- Desbellote: Se elimina la flor o bellota del racimo.

- Desmane: Se retiran las manos que no alcancen los requisitos para la comercialización.

- Deshoje: consiste en eliminar las hojas maduras, dobladas y/o secas que existan en el racimo para que no interfiera en su desarrollo. 
- Desflore: se retiran los residuos florales para prevenir plagas y enfermedades que provoquen daños futuros en la producción.

- Embolse: consiste en colocar una bolsa plástica en cada racimo la cual crea un microclima idóneo para su desarrollo, también protege al fruto de insectos u otros animales.

- Encintado: cada racimo lleva una cinta de diferente color el cual permite conocer la edad del racimo y determinar su punto óptimo para la cosecha.

- Deshijado: se retiran los hijos y se vuelven a sembrar después de cada corte.

- Recepción de fruta: es el área en donde se depositan los racimos que serán procesados.

- Desleche y lavado: en la tina se aplica un removedor de látex, se transportan los clústeres por un flujo de agua para ser seleccionados y clasificados la fruta que cumplan los requisitos de comercialización.

- Pesado: se pesa en una balanza calibradora el número de clúster necesarios para conseguir el peso de la caja a comercializar.

- Tratamiento: se aplica un producto que contenga sulfato de aluminio y fungicida en la corona para evitar la pronta descomposición del fruto durante el almacenamiento y transporte, también previene el desarrollo de hongos.

- Etiquetado: son distintivos de las marcas según el destino a donde serán transportadas, se ubican en los dedos interiores del clúster.

- Empaque: la caja cuenta con las dimensiones idóneas para el peso a transportar, el fruto se ubica en tres o cuatro filas según el tamaño del mismo, bajo separadores y un papel absorbente en la base de la caja de cartón, la misma lleva una tapa con orificios ideal para la ventilación.

- Embalaje: esta actividad se lleva a cabo en área de empaque, se procede a realizar la carga de las cajas procesadas, las cuales se colocan en pallet para su transportación.

Según INEC (2021), De la producción nacional en banano la provincia de El Oro representa el 24,59\%, aunque no lidera en nivel productivo, sus unidades producidas la ubican entre la más productiva, cuenta con la mayor cantidad de haciendas registradas en la Subsecretaría de Comercialización del Ministerio de Agricultura, sin embargo, la mayor parte está conformada de pequeños y medianos productores, los cantones dedicados a la producción de banano son: Machala, Santa Rosa, El Guabo, Pasaje y Arenillas, permite que se involucren alrededor de 100 mil trabajadores de forma directa e indirecta. 
Figura 3. Producción de banano en la Provincia de El Oro.

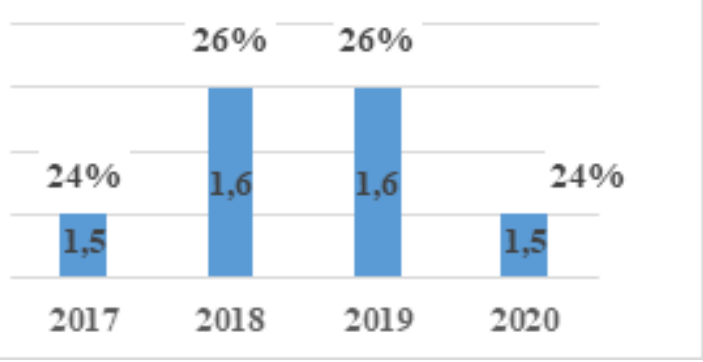

Fuente: INEC (2021)

En la Figura 3, se aprecia la producción de banano dentro de la provincia de El Oro con un total de 6.2 miles de ${ }^{\mathrm{TM}}$ en los últimos 4 años, se evidencia un crecimiento en el transcurso del tiempo, sin embargo, en el año 2020 la emergencia sanitaria de 1 COVID-19 causó un notable descenso en la producción pese a su participación del $27 \%$ en la superficie plantada, ocasionado por las dificultades logísticas en el traslado de la fruta, siendo similar al rendimiento obtenido en el año 2017, en la provincia la economía se vio afectada significativamente debido a su participación.

\section{METODOLOGÍA}

Para desarrollar el presente trabajo se empleó una metodología bajo un diseño no experimental de campo y corte transversal con enfoque descriptivo-explicativo de los datos obtenidos en la Hacienda bananera "San Alfonso" ubicada en el sitio la Bocatoma del cantón el Guabo, provincia de El Oro, la cual cuenta con una extensión de 35,63 hectáreas dedicadas a la producción de banano variedad Cavendish.

Al recabar información de la producción en el periodo 2018-2019 se procede a tabular los datos a través del programa Microsoft Excel, se utilizó una estadística descriptiva con el fin de analizar los indicadores productivos para conocer el año con mayor productividad, véase en la Tabla 2.

Tabla 2 Indicadores

Productivos Económicos

Cajas procesadas: El nivel de cajas Ingresos: Entradas monetarias obtenidas de las obtenidas por hectárea refleja la eficiencia del ventas, contribuye en el crecimiento del patrimonio área a producir y su rentabilidad. de la empresa.

Ratio: relación entre la cantidad total de Egresos: Salidas de dinero, disminuyen los racimos cosechados con el número de cajas producidas, depende del vigor y la calidad del racimo (Villaseñor, et al., 2020). beneficios, en el caso de los costos son necesarios para producir mientras que los gastos son necesarios para financiar.

Recobro: Control en la edad de cada racimo Utilidad: Es utilizado para determinar el de banano plantado en una superficie comportamiento económico, representa las determinada, identificados con cintas de ganancias o pérdidas obtenidos (Ordoñez, et al., diferentes colores (Torres, et al., 2013). 2019). 
Merma: es el producto rechazado dentro de las hectáreas productivas, ya sea por golpes, manchas o por incumplir con los lineamientos para la comercialización (Colque , 2017).
Punto de equilibrio (U): Determina el nivel de unidades producidas para equilibrar los gastos, su fórmula es costo de producción entre el precio a comercializar (Mazón, et al., 2017).
Fruta exportable: peso de la fruta aprovechada en la cosecha, es un indicador de rendimiento (Vásquez, et al., 2019).
Beneficio/Costo: determina la viabilidad, siempre y cuando el índice es mayor a 1 , es el beneficio generado por cada unidad producida en términos financieros (Garzón, et al., 2019).

\section{RESULTADOS Y DISCUSIÓN}

Figura 4. Producción semanal de cajas de banano en la Hacienda San Alfonso, periodo 2018-2019.

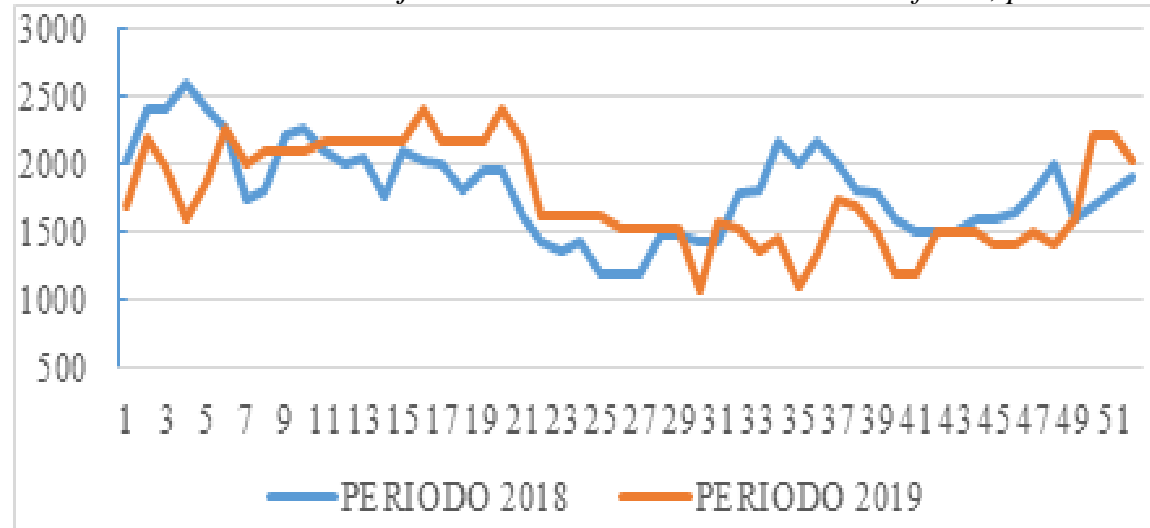

Fuente: Productor Agrícola el Cocoke S.A "Hacienda San Alfonso”

En la figura 4. se observa un menor rendimiento productivo en el año 2018 entre los meses de junio hasta agosto, sufre un retroceso en la semana 38, el cual se extiende a la semana 41 correspondiente al mes de octubre, mientras que en el 2019 va desde junio a noviembre, este descenso se denota influenciado por el factor clima, temporada de verano la cual tiene una temperatura seca y fresca, causando un daño significativo a las plantaciones de banano, puesto que no les permite desarrollarse como corresponde, debido a que necesitan numerosas horas de luz solar por día para una mejor producción, con una diferencia de 2.843 cajas entre ambos periodos.

Tabla 3 Análisis de Indicadores Productivos

\begin{tabular}{|c|c|c|}
\hline Productividad & 2018 & 2019 \\
\hline Cajas & 94295 & 91452 \\
\hline Enfunde a cosechar & 83408 & 85288 \\
\hline Racimos cosechados & 81304 & 82521 \\
\hline
\end{tabular}




\begin{tabular}{|c|c|c|}
\hline Racimos rechazados & 788 & 1000 \\
\hline Peso de racimos & 1457,6 & 1407,3 \\
\hline Ratio & 1,16 & 1,11 \\
\hline Recobro & $97,47 \%$ & $96,77 \%$ \\
\hline Fruta aprovechada & $79,31 \%$ & $77,96 \%$ \\
\hline Merma & $20,69 \%$ & $22,04 \%$ \\
\hline
\end{tabular}

Fuente: Productor Agrícola el Cocoke S.A "Hacienda San Alfonso"

En la tabla 3. De acuerdo a lo manifestado por Durán (2011), sobre el nivel óptimo de merma y recobro aceptable en la producción de banano, podemos percibir en función del recobro entre los racimos enfundados y los racimos a cosechar, está dentro del rango óptimo, el cual va desde el 95\% al 100\%, sin embargo, la calidad de los racimos obtenida en la producción es baja en relación al peso, por ello, el nivel de la fruta cuya característica no cumple con los requisitos para la comercialización en el mercado exterior supera el $11 \%$ al $16 \%$ de merma aceptada, lo cual influye directamente con el rendimiento productivo de la hacienda.

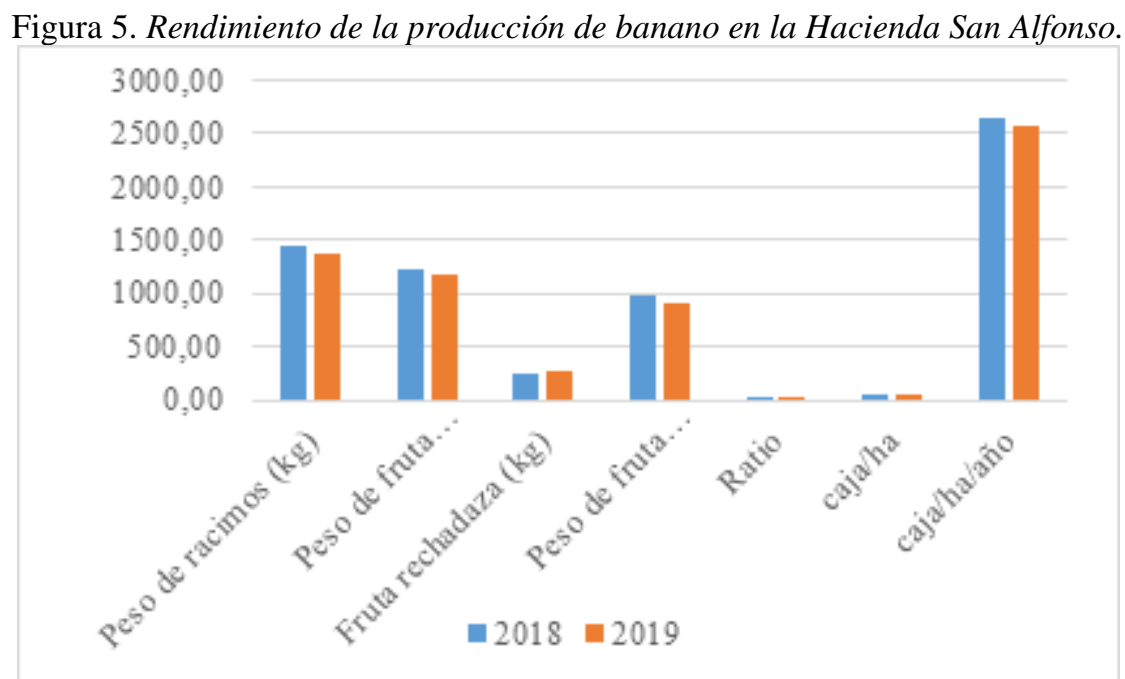

Fuente: Productor Agrícola el Cocoke S.A "Hacienda San Alfonso"

De los datos obtenidos en la tabla 3, se analiza que del total en el peso de los racimos cosechados por las 35,63 ha, en el año 2018 el peso promedio de la fruta cosechada es de 1231, con una merma de 255 la cual representa el 20,16\%, lo que significa que la fruta para la exportación es de 976 o un 79,84\%, mientras que en el año 2019 es de 1179 del cual 260 o el 23,29\% fue el rechazo, por ende, la fruta para la exportación es de 919 o el 76,71\%. 
Tabla 4 Análisis de Indicadores Económicos

\begin{tabular}{|c|c|c|}
\hline \multirow[t]{2}{*}{ CUENTAS } & \multicolumn{2}{|c|}{ SALDOS } \\
\hline & 2018 & 2019 \\
\hline (+) Ingresos & $587.452,53$ & $606.866,82$ \\
\hline (+) Ingresos varios & $3.499,92$ & $3.500,00$ \\
\hline (-) Costos de producción & $410.865,66$ & $419.331,63$ \\
\hline (-) Gastos & $146.109,33$ & $159.070,30$ \\
\hline (-) Gastos no deducibles & 411,52 & 316,53 \\
\hline (=) Utilidad del Ejercicio & $33.565,94$ & $31.648,36$ \\
\hline Beneficio/Costo & 1,43 & 1,45 \\
\hline Precio de comercialización & 6,23 & 6,64 \\
\hline $\begin{array}{l}\text { Punto de equilibrio en unidades } \\
\text { producidas /año }\end{array}$ & 65.950 & 63.191 \\
\hline
\end{tabular}

Fuente: Productor Agrícola el Cocoke S.A "Hacienda San Alfonso"

En la tabla 4. Una vez realizado el análisis financiero, tenemos un costo de producción por cada caja de banano de $\$ 4,35$, en función a la relación beneficio-costo, este nos indica que por cada dólar invertido se obtiene 0,43 centavos de ganancia, dado que el precio de comercialización es de \$6,23 en el año 2018, mientras que en 2019 el costo de producción por cada caja de banano es de $\$ 4,58$, con un retorno de 0,45 centavos de ganancia por cada dólar invertido y un precio de comercialización de \$6,64.

Según Martínez, et al., (2015), el punto de equilibrio en unidades producidas, donde el costo total se divide para el precio de comercialización, indica el efecto positivo o negativo si la productividad es mayor o menor al resultado calculado, al aplicar la fórmula, el total de cajas producidas están por encima de las unidades a producir tanto en el año 2018 como en el año 2019, indica la eficiencia operacional del volumen productivo dado que el nivel de ingreso por las cajas vendidas cubren los costos y cada unidad adicional representa una ganancia.

\section{CONCLUSIÓN}

En la actividad bananera la producción de cajas indica el rendimiento productivo, sin embargo, las variables económicas determinan la ganancia o pérdida en el año, por lo tanto se señala la relación directa entre los indicadores productivos y económicos, dado que en la Hacienda San Alfonso el mayor número de cajas procesadas por año, se obtuvo en el periodo 2018 con un total de 94.295 cajas, generando un ingreso económico de \$587.452,53, mientras que en el año 2019 el nivel de ingreso es de $\$ 606.866,82$ por 91.452 cajas procesadas, se debe a la variación constante en el precio, en relación al año anterior es superior con 0,40 centavos, jugando un papel fundamental, no obstante, el año más productivo de la 
Hacienda bananera es el periodo 2018 en términos agrícolas asociados con la producción, de acuerdo al indicador ratio por cada racimo cortado se obtiene 1,16 cajas procesadas, con el cual se evidencia mayor cantidad de fruta exportada y menor cantidad de rechazo, es decir, se logró un mejor manejo dentro del sistema productivo, del cual se obtuvo una utilidad positiva de $\$ 33.565,94$. 


\section{REFERENCIAS}

BANCO CENTRAL DEL ECUADOR. (octubre de 2019). https://contenido.bce.fin.ec/. ISSN:1390-0579 Chica Guerrero, M. B., López Fernández, R., Medina Peña, R., \& González Romero, J. C. (mayo de 2020). Caracterización en cultura medio ambiental de las bananeras en la provincia de El Oro. Revista Metropolitana de Ciencias Aplicadas, 3(2), 216-224. doi:2631-2662

Chungata Campoverde, A. E., Chungata Jiménez, K. E., Vite Cevallos, H. A., \& Barrezueta Unda, S. (mayo de 2019). Perfil socioeconómico y ambiental de la Asociación Agraria Bananera Fincas de El Oro. Revista Metropolitana de Ciencias Aplicadas, 2(2), 182-190. doi: 2631-2662

Colque, O. (2017). Rendimiento y calidad de fruta de cuatro clones de banano (Musa aaa) en el subtrópico de la Provincia Formosa, Argentina. Revista Bolivarianas, 4(9), 15.21. doi:2308-250X

Durán Mera, C. A. (2011). www.dspace.espol.edu.ec. Obtenido de https://www.dspace.espol.edu.ec/retrieve/100062/D-79555.pdf

FAO. (noviembre de 2020). http://www.fao.org/. Obtenido de http://www.fao.org/fileadmin/templates/est/COMM_MARKETS_MONITORING/Tropical_Fruits/Docu ments/Commodity_Focus_FO_NOV_2020_SP.pdf

FAO. (noviembre de 2020). http://www.fao.org/. Obtenido de http://www.fao.org/3/ca9212es/ca9212es.pdf

Galarza Suárez, L. (2019). Tierra, trabajo y tóxicos: sobre la producción de un territorio bananero en la costa sur del Ecuador. Estudios atacameños (63). doi:http://dx.doi.org/10.22199/issn.0718-1043-20190034

García Saltos, M. B., Juca Maldonado, F., \& Juca Maldonado, O. M. (2016). ESTUDIO DE LOS ESLABONES DE LA CADENA DE VALOR DEL BANANO EN LA PROVINCIA DE EL ORO. Universidad y Sociedad [online], 8(3), 51-57. doi:2218-3620

Garzón Montealegre, V. J., Ramírez Morales, I., Coronel Reyes, J., \& Serrano Ruge, B. H. (mayo de 2019). Determinación de la factibilidad del uso de espectrometría portátil para el control de calidad en materias primas utilizadas en panaderías y pastelerías en la ciudad de Machala, Ecuador. Revista Metropolitana de Ciencias Aplicadas, 2(2), 141-150. doi: 2631-2662

INEC. (mayo de 2020). ecuadorencifras.gob.ec. Obtenido de https://www.ecuadorencifras.gob.ec/documentos/web-inec/Estadisticas_agropecuarias/espac/espac2019/Presentacion\%20de\%201os\%20principales\%20resultados\%20ESPAC\%202019.pdf

INEC. (mayo de 2021). https://www.ecuadorencifras.gob.ec/. Obtenido de https://www.ecuadorencifras.gob.ec/documentos/web-inec/Estadisticas_agropecuarias/espac/espac2020/Presentacion\%20ESPAC\%202020.pdf

Lassoudière, A. (2010). L'HISTOIRE DU BANANIER (1a ed.). Éditions Quae. Libro electrónico EAN13 [PDF]: 9782759206193 
Martínez Medina, I., Val Arreola, D., Tzintzun Rascón, R., Conejo Nava, J. d., \& Tena Martínez, M. J. (abril-junio de 2015). Competitividad privada, costos de producción y análisis del punto de equilibrio de unidades. Revista Mexicana de Ciencias Pecuarias, 5(2), 193-205. ISSN: 2007-1124

Mazón Arevalo, L., Villao Burgos, D., Nuñez, W., \& Serrano Luyó, M. (junio de 2017). Análisis de punto de equilibrio en la toma de decisiones de un negocio: caso Grand Bazar Riobamba -Ecuador. Revista de Estrategias del Desarrollo Empresarial, 8, 14-24. Obtenido de: https://www.ecorfan.org/spain/researchjournals/Estrategias_del_Desarrollo_Empresarial/vol3num8/Revi sta_de_Estrategias_del_Desarrollo_Empresarial_V3_N8_2.pdf

Ministerio de Comercio Exterior. (diciembre de 2017). https://www.produccion.gob.ec/. Obtenido de https://www.produccion.gob.ec/wp-content/uploads/2019/06/Informe-sector-bananero-espa\%C3\%B1ol04dic 17.pdf

Ordoñez, J., Vite Cevallos, H., \& Barrezueta Unda, S. (mayo de 2019). Análisis de rentabilidad económica del plátano (Musa balbisiana $\mathrm{AAB}$ Simmond) en el sitio Río Negro provincia El Oro. Revista Metropolitana de Ciencias Aplicadas, 2(2), 160-170. doi:2631-2662

Prado Carpio, E., Quiñonez Cabeza, M., Rentería Minuche, P., Maetínez Soto, M., Rodríguez Monroy, C., \& Olivo Garrido, M. (6 de junio de 2021). Estándar de Calidad del Molusco Bivalvo "Concha Prieta" (Anadara Tuberculosa). 19th LACCEI International Multi-Conference for Engineering, Education, and Technology. doi:10.18687 /LACCEI 2021.1.1.443

Torres Rodríguez, A., Bernal Vera, M. E., \& Castaño Ramírez, E. (enero-junio de 2013). EVALUACIÓN AMBIENTAL DE LA PRÁCTICA “EMBOLSADO” EN PLÁTANO (Musa AAB SIMMONDS). QUINDÍO, COLOMBIA. Revista Luna Azul (36), 91-109. doi:1909-2474

Vásquez Castillo, W., Racines Oliva, M., Moncayo, P., Viera, W., \& Seraquive, M. (octubre de 2019). Calidad del fruto y pérdidas poscosecha de banano orgánico (Musa acuminata) en el Ecuador. Enfoque UTE, 10(4), 57-66. doi:https://doi.org/10.29019/enfoque. v10n4.545

Villaseñor, D., Noblecilla Romero, Y., Luna Romero, E., Molero Naveda, R., Barrezueta Unda, S., Huarquila Henriquez, W., . . Garzón Montealegre, J. G. (2020). RESPUESTA ÓPTIMA ECONÓMICA DE LA FERTILIZACIÓN POTÁSICA SOBRE VARIABLES PRODUCTIVAS DEL BANANO (Musa spp.). AgroCiencia, 36(2), 161-170. doi:https://doi.org/10.29393/CHJAAS36-14RODV80014

Vitali, S. (enero-junio de 2017). Precariedad en las condiciones de trabajo y salud de los trabajadores del sector bananero del Ecuador. Salud de los Trabajadores, 25(1), 9-22. doi:1315-0138

Zhiminaicela Cabrera, J. B., Quevedo Guerrero, J. N., \& García Batista, R. M. (septiembre de 2020). La producción de banano en la Provincia de El Oro y su impacto en la agrobiodiversidad. Revista Metropolitana de Ciencias Aplicadas, 3(3), 189-195. doi:2631-2662 